\title{
Overnight Delta Dynamics Associated with Daytime Psychomotor Performance in Adults with Insomnia and Healthy Controls
}

\author{
Jessica R Lunsford-Avery (D)', Jack D Edinger ${ }^{1,2}$, Andrew D Krystal ${ }^{1,3}$ \\ 'Department of Psychiatry and Behavioral Sciences, Duke University School of Medicine, Durham, NC, 277I0, USA; ${ }^{2}$ Department of Medicine, \\ National Jewish Health, Denver, CO, 80206, USA; ${ }^{3}$ Departments of Psychiatry and Neurology, University of California San Francisco School of \\ Medicine, San Francisco, CA, 94I43, USA
}

Correspondence: Jessica R Lunsford-Avery, Department of Psychiatry and Behavioral Sciences, Duke University School of Medicine, 2608 Erwin Road Suite 300, Durham, NC, 27710, USA, Tel + 919-681-0035, Fax + 1919-681-0016, Email jessica.r.avery@duke.edu

Purpose: Sleep is vital to cognition, yet underlying mechanisms remain unclear. Although sleep duration and continuity are two wellestablished contributors, additional factors — including homeostatic sleep drive processes — may also underlie cognition-related sleep restoration. This study investigates the relative contributions of sleep EEG factors to psychomotor functioning in adults with insomnia and healthy controls (HC) to identify the most significant sleep factors supporting psychomotor functioning.

Materials and Methods: Adults with insomnia $(n=37)$ and HC $(n=39)$ completed 3 nights of polysomnography and a complex psychomotor task (switching attention task; SAT). Univariate correlations identified the most significant predictors (traditional PSG, spectral EEG, initial delta peak, and overnight delta decline) of SAT performance, which were then entered into multivariable linear regressions examining whether predictors remained significant after accounting for shortened/fragmented sleep and whether relationships differed across groups.

Results: In addition to greater wake after sleep onset (WASO; $r=0.33$ ), a slower overnight delta decline $(r=0.50)$ and a lower initial delta peak $(r=-0.38)$ were the most significant predictors of poorer SAT performance. Both overnight delta decline $(\mathrm{F}(7,68)=12.52$, $p<0.001)$ and initial delta peak $(\mathrm{F}(7,68)=7.85, p=0.007)$ remained significant predictors after controlling for demographics, total sleep time, and WASO. Relationships were analogous across subject groups.

Conclusion: Findings suggest that, in addition to sleep duration and continuity, processes related to recovery from and dissipation of homeostatic sleep drive may support psychomotor performance and broadly support daytime functioning in individuals with and without insomnia. Future research may examine overnight delta dynamics as transdiagnostic processes supporting cognition-related sleep restoration across a range of clinical populations.

Keywords: insomnia, EEG, homeostatic sleep drive, delta power, cognition, reaction time

\section{Plain Language Summary}

Although sleep duration and continuity are well-established contributors to cognitive function, additional sleep factors may also play vital roles. This study is the first to demonstrate relationships between processes related to homeostatic sleep drive (ie, the pressure to sleep that builds the longer a person is awake and dissipates during sleep) and psychomotor performance in adults with and without insomnia disorder after accounting for the effects of sleep duration and continuity. Findings suggest that processes related to recovery from and dissipation of homeostatic sleep drive may support psychomotor functioning, and have broad clinical implications for adults with psychomotor impairments. In addition, results suggest that interventions known to improve homeostatic sleep drive such as cognitive-behavioral therapy for insomnia may support psychomotor functioning in adults.

\section{Introduction}

Sleep is critical for supporting cognition and daytime functioning. ${ }^{1}$ However, the underlying mechanisms by which sleep supports cognition are unclear. Prior work using sleep deprivation paradigms has shown that experimentally reducing 
sleep time in normal sleepers adversely impacts cognition, and that daytime functioning is restored by the physiology underlying "recovery sleep". ${ }^{1-3}$ This literature suggests a process of sleep restoration supporting daytime cognition (ie, a neurobiological process in which the brain functions necessary for cognition are rejuvenated during sleep ${ }^{4}$ ), but does little to elucidate the specific aspects of sleep that are necessary for supporting cognitive functioning when normal sleep occurs without experimental restriction.

Two lines of research have revealed daytime impairments that occur when sleep is disturbed but not experimentally curtailed. First, studies using experimental sleep fragmentation methods have shown that intermittently interrupting sleep with sensory (eg, auditory, tactile) stimuli results in next-day cognitive impairment, sleepiness, and mood changes, ${ }^{5,6}$ suggesting that such disturbances reduce the restorative capacity of sleep even in the absence of total sleep time (TST) reductions. In addition, sleep fragmentation appears to interrupt the sleep restoration process and hinder cognitive performance even when brief awakenings do not result in full waking consciousness. ${ }^{7}$ However, not all studies of sleep fragmentation have shown associations with cognitive deficits, ${ }^{8,9}$ suggesting that daytime impairment resulting from interrupted sleep may be more subtle and less universal than deficits observed under sleep deprivation conditions. ${ }^{5}$

Second, investigations with adults with insomnia disorder have suggested that affected individuals report they feel sleepy, fatigued, and cognitively impaired. These daytime deficits are consistently reported even though up to $40 \%$ of adults with insomnia disorder do not display reductions in TST as measured by polysomnography (PSG). ${ }^{10}$ However, studies with adults with insomnia do not consistently find cognitive deficits as measured by objective methods, such as the simple reaction time (SRT) and continuous performance tests (CPT) ${ }^{11-16}$ This apparent inconsistency in findings may be due to the use of small samples as well as the employment of cognitive tests that are limited in the degree to which they challenge cognitive capacity. ${ }^{15}$ For example, longer reaction times have been observed in patients with insomnia compared to controls on an attentional task, but only under conditions of high cognitive load, ${ }^{17}$ and a recent metaanalysis suggests that tasks associated with higher sensitivity, such as attention-switching tasks, are necessary to detect the executive functioning deficits characteristic of insomnia. ${ }^{18}$

Prior work completed by our group addressed these limitations by examining PSG-measured sleep disturbances and next-day cognitive functioning in a large sample (79 patients with insomnia, 84 healthy controls) incorporating a more challenging test of psychomotor performance (switching attention test, SAT). ${ }^{15}$ This study found that although adults with insomnia and healthy controls did not differ in their performance on the less challenging psychomotor tasks (CPT and SRT), patients with insomnia performed significantly worse on the more demanding task (SAT). Notably, this difference in psychomotor performance on the SAT was not due to sleep deprivation, as the two groups did not differ in TST. Rather, poorer cognitive functioning was most strongly predicted by greater wake after sleep onset (WASO) for the entire sample as well as for each group separately, suggesting that sleep continuity may be a universal process underlying cognition-related sleep restoration.

Although sleep continuity may be one component of the sleep restoration process supporting cognition, it is likely that additional sleep factors contribute to daytime cognitive functioning that were not addressed by this prior study. First, investigations using sleep deprivation methods have suggested that restoration may be related to the initial elevation in EEG-measured delta amplitude (initial delta peak) and its rate of decline overnight (delta decline). Specifically, a large body of evidence indicates that initial delta peak increases and delta declines becomes steeper with waking duration and are reduced by manipulations that reduce the period of prior waking (eg, naps), supporting these indices as indicative of "homeostatic sleep drive" and the degree of restoration that occurs during sleep. ${ }^{19-32}$ In addition, delta decline is shown in animal models to have identifiable neurobiological substrates, and when there is dysfunction in those neurobiological processes in humans it leads to cognitive deficits. ${ }^{33-40}$ However, it is currently unknown whether this phenomenon is unique to post-deprivation recovery or is integral to the restorative process of normal sleep when it is unrestricted. In addition, our recent work has shown lower initial delta power peak and a slower decline in delta power overnight among patients with insomnia compared to healthy controls, ${ }^{41}$ highlighting these as potential factors of interest for understanding cognitive deficits in this population.

Second, an extensive literature has suggested that decreased sleep spindle density (reflected by lower sigma power) is related to cognitive impairment as well, ${ }^{42-50}$ highlighting another potential component of the cognition-related sleep restoration process. Finally, recent research examining a range of sleep EEG predictors has suggested that greater slow 
wave sleep (SWS) may support faster response time, while sleep continuity and more rapid eye movement (REM) sleep may support accuracy, on cognitive tasks among healthy adults without sleep complaints, ${ }^{51}$ suggesting additional potential measures worthy of further investigation.

In the current study, we extended our prior work examining traditional PSG indices and daytime psychomotor performance in adults with insomnia and healthy controls to include EEG spectral power indices as well as calculations of initial delta peak and overnight delta decline. Our primary objective was to investigate the relative contributions of these sleep factors to complex attention, one indicator of daytime psychomotor functioning, on a complex psychomotor task (SAT) and to identify the most significant indices supporting the cognition-related sleep restoration process. We examined these relationships in healthy controls in addition to in adults with insomnia to provide a context for understanding any observed relationships between sleep factors and psychomotor functioning in our insomnia sample. As mentioned above, we have previously shown poorer SAT performance among adults with insomnia compared to healthy controls ${ }^{15}$ however, whether relationships between sleep factors and psychomotor functioning are specific to insomnia or reflect a broad restoration process cutting across individuals with and without insomnia is unknown.

Based on the reviewed literature, we hypothesized two sleep-based contributors to daytime cognitive functioning other than sleep duration and continuity, including: 1) accumulation of homeostatic sleep drive (reflected by initial delta peak) and its dissipation over the sleep period (reflected by overnight delta decline); and 2) sigma power (as it is associated with sleep spindles). Further, we hypothesized that these sleep factors would be associated with psychomotor functioning after accounting for sleep duration and continuity. In addition, as we believe these indices may reflect a universal cognition-related sleep restoration process, we anticipated that similar processes would support psychomotor performance in both the insomnia disorder and healthy control groups. Finally, to examine whether analogous processes support response time across psychomotor tasks, we explored sleep EEG contributors to performance on commonly used psychomotor tasks (CPT and SRT). Although our primary focus was SAT performance, given prior literature suggesting greater insomnia-related impairment on more complex cognitive tasks, ${ }^{15,17,18}$ if similar sleep factors are associated with better performance on CPT and SRT tasks, this would provide additional support for a potential role of those specific sleep factors in supporting psychomotor functioning.

\section{Materials and Methods}

\section{Participants}

The current sample was drawn from a series of prior studies examining home- and lab-measured sleep patterns of adults with insomnia disorder (aged 20-80) and age- and gender-matched healthy controls (HC) without sleep complaints. ${ }^{15,52-}$ ${ }^{54}$ Recruitment strategies included posted advertisements as well as written and face-to-face solicitations of individuals in Duke's Center for the Study of Aging and Human Development Subject Pool or presenting to Duke's sleep disorders clinic. Study procedures were in accordance with the Declaration of Helsinki and were approved by the Institutional Review Boards of Duke University Medical Center and VA Medical Center in Durham, NC. Participants provided informed consent and were financially compensated for their time and costs (eg, parking) associated with study participation.

To assess eligibility for the study, individuals completed a screening visit which included structured psychiatric ${ }^{55}$ and sleep ${ }^{56}$ interviews and a medical exam including a thyroid (TSH) level screening (an exclusionary criterion, due to likely impact of hypothyroidism on sleep indices). In addition, participants completed 1 night of PSG to detect occult primary sleep disorders (another exclusionary criterion). Included individuals in the insomnia group met research diagnostic criteria for insomnia disorder (ie, at least 6 months of problems with initiating sleep, maintaining sleep, or nonrestorative sleep accompanied by daytime impairment). ${ }^{57}$ Inclusion criteria for healthy controls included medically and psychiatrically healthy adults devoid of sleep complaints and sleep disorders.

Potential participants were excluded if presenting with: a medical condition disturbing sleep, current axis I psychiatric disorder, sedative hypnotic dependence, current use of psychotropic medications, or significant symptoms of sleep apnea (apnea-hypopnea index $[\mathrm{AHI}] \geq 5$ ) or periodic limb movement disorder (PLM-related arousal index [PLMAI] $\geq 5$ ) as assessed by the PSG screening. AHI and PLMAI exclusion criteria were stricter than typically employed in sleep 
research studies ${ }^{15,44,51}$ in order to prevent the detection of sleep indices impacting psychomotor functioning that are related to sleep-related breathing disorders or periodic limb movements of sleep, which are already well-established factors impairing sleep restoration underlying cognition. ${ }^{58,59}$ In addition, to minimize spurious findings due to inadequate sampling of data while maximizing power to detect effects, we included in the analysis only sleep studies that had nonREM data that was artifact-free for at least $10 \%$ of TST. Rationale for and data supporting this cutoff have been previously described. ${ }^{41}$ Of 163 participants in our prior study, ${ }^{15} 128$ participants provided sleep studies that were available and suitable for spectral analysis. Of these, 76 participants (37 in the insomnia group and $39 \mathrm{HC}$ ) met the aforementioned criteria for inclusion in current analyses. The data underlying this article will be shared on reasonable request to the senior author (ADK).

\section{Polysomnography}

Participants completed 3 nights of either lab-based or home-based PSG recording directly prior to psychomotor assessment. PSG location (lab- or home-based) was randomly assigned such that one-half of the men and women in both groups completed lab-based PSG while the other half underwent home-based PSG. All participants, regardless of PSG location, were asked to maintain their customary bedtimes and rising times during participation. PSG data were recorded using 8-channel Oxford Medilog 9000 or 9200 series ambulatory cassette devices (Oxford Medical Inc., Tampa, Fl.) and a standard monitoring montage including 2 electroencephalogram (EEG) channels $\left(\mathrm{C}_{3}-\mathrm{M}_{2}, \mathrm{O}_{\mathrm{z}}-\mathrm{C}_{\mathrm{z}}\right)$, bilateral electrooculogram (EOG), submental electromyogram (EMG), 2 channels of anterior tibialis EMG (right and left leg), and a nasal-oral thermistor. ${ }^{60,61}$

An Oxford Vision System (Oxford Instruments Inc., Tampa, Fl.) was employed to digitize the EEG data at $128 \mathrm{~Hz}$ with 8-bit accuracy. Filter settings of 0.5 and $64 \mathrm{~Hz}$ were used with $-3 \mathrm{~dB}$ roll-off at both ends and a third-order Butterworth antialiasing filter at $64 \mathrm{~Hz}$. Data were scored by coauthor ADK (Board-Certified in Sleep Medicine, blind to group status) in 30-second epochs. Standard scoring criteria were employed to assign sleep stages, ${ }^{10,62}$ identify respiratory (eg, apneas, hypopneas) and PLM-related arousals during the first night of recording (exclusionary criteria), and calculate mean values (across 3 nights of recording) for time in bed (TIB), total sleep time (TST), sleep onset latency (SOL), wake time after sleep onset (WASO), and sleep efficiency (SE).

\section{EEG Analysis}

As is consistent with our prior work, ${ }^{41,63}$ spectral analysis was conducted on a single $\mathrm{C}_{3}-\mathrm{M}_{2}$ (left central to right mastoid) channel, and all included epochs were verified to be free of movements, artifacts, arousals, and sleep stage transitions based on visual review and manual artifacting by coauthor ADK, who is Board-Certified in Sleep Medicine and Clinical Neurophysiology and highly experienced in spectral analysis of non-REM sleep EEG data. ${ }^{10,63,64}$ Autoregressive highpass filtering and Hanning windowing were applied to EEG data followed by fast Fourier transformation in 2-second epoch averaging over time and frequency. This analysis was conducted using custom software written in Visual $\mathrm{C}++$ by ADK, which employed MATLAB (The MathWorks, Inc., Natick, MA), and yielded 6 frequency bands: delta (0.5-3.5 $\mathrm{Hz}$ ), theta $(4.0-8.0 \mathrm{~Hz})$, alpha $(8.5-12 \mathrm{~Hz})$, sigma $(12.5-16 \mathrm{~Hz})$, beta $(16.5-30 \mathrm{~Hz})$, and gamma $(30.5-60 \mathrm{~Hz})$. The bands were chosen based on prior work conducted by our group and others and were verified as described in our earlier papers. $^{10,65-67}$

Consistent with prior research, mean values for relative spectral power were calculated by computing power of each sleep stage in $\mu \mathrm{V}^{2}$ overnight, dividing the power in each band overnight by the total sum of power across all bands, and averaging across the 3 nights of data. ${ }^{10,65-67}$ We elected to average across 3 nights of data due to prior research suggesting that a single night of PSG recording is not sufficient for adequate stability of sleep parameters in individuals with insomnia or healthy controls (in either lab or home settings), and, as a result, averaging over multiple nights is necessary to reduce the influence of random variations. ${ }^{68}$ Based on our prior study demonstrating differences in overnight delta power between individuals with insomnia and $\mathrm{HC}$, we also computed initial delta power peak (delta power at the time of sleep onset) and the decline of delta power overnight by fitting a linear best-fit line using the least-squares method. This computational method was selected as it has been shown to be less vulnerable to missing data that occur as a result of artifacts, state transitions, etc. ${ }^{41}$ 


\section{Psychomotor Performance Assessment}

Psychomotor performance was assessed using the computer-based Neurobehavioral Evaluation System (NES). ${ }^{69}$ The NES was conducted in the lab-setting approximately $2-3$ hours following the individual's rising time. The NES was administered in four 16-minute trials, and each trial consisted of a series of three reaction time tasks that ranged in difficulty from least challenging (simple reaction time test; $\mathrm{SRT}^{11,12}$ ) to moderately challenging (continuous performance test; $\mathrm{CPT}^{13}$ ) to most challenging (switching attention task; $\mathrm{SAT}^{11,70}$ ). Participants were individually assessed via PC computer in a quiet testing room. Accompanying NES software provided task instructions, administered test items, and recorded participant responses for each trial of each test (ie, milliseconds (ms) elapsed between onscreen stimulus presentation and computer key-press response).

Each trial initiated with the SRT, which required participants to press an assigned key on the keyboard each time the target stimulus (ie, a small square) appeared on the screen. Stimuli were presented at intervals ranging from 1000 to 2500 $\mathrm{ms}$ and remained onscreen until a response was made or $1000 \mathrm{~ms}$ elapsed. Each SRT trial consisted of 90 presentations of the target stimulus and lasted approximately 5 minutes. Subsequent to the SRT, participants completed the CPT which presented both target (ie, the letter S) and distractor (ie, letters A, C, E, and T) stimuli on the computer screen at a rate of 1 per second. Each stimulus remained onscreen for $50 \mathrm{~ms}$, and stimuli were presented in random order with a 1:4 targetto-distractor ratio. Participants were asked to press the assigned key when (and only when) the target stimulus appeared on the screen. Each 5-minute CPT trial comprised 60 target stimuli and 240 distractor stimuli.

Finally, the complex SAT consisted of three sequential reaction time subtests. Throughout the SAT, participants were asked to respond to presented stimuli by pressing a mark key on the right or left side of the keyboard. During the first segment of the SAT, a target stimulus (ie, a square) was presented on the right or left side of the screen and participants were requested to press the key on the corresponding side of the keyboard. This task consisted of 6 practice and 16 test presentations per trial. In the second segment, a target stimulus (ie, an arrow pointing left or right) was presented in the middle of the screen and participants were instructed to press the side key that corresponded to the direction of the arrow. This task consisted of 4 practice and 16 test presentations per trial. For each of these tasks, stimuli remained onscreen until a response was provided or $2500 \mathrm{~ms}$ elapsed.

During the final, most challenging segment of the SAT, participants viewed arrows on the right or left side of the screen, which were pointing left or right. One-thousand ms prior to presentation of an arrow, a command word ("SIDE" or "DIRECTION") was presented, which directed participants to press a key on the side of the keyboard corresponding to either 1) the side of the screen on which the arrow appeared or 2) the direction the arrow was pointing. These stimulus characteristics agreed on $50 \%$ of trials and conflicted on $50 \%$ of trials and were presented in a random sequence. This final segment consisted of 8 practice and 48 test presentations per trial. Poor performance on each of the NES subtests (ie, SRT, CPT, and SAT) has been shown to be reliable, valid detectors of subtle cognitive impairment. ${ }^{11,12,70}$ However, prior research from our group has suggested that the SAT may be particularly sensitive to detecting cognitive impairments resulting from sleep disturbances. ${ }^{15}$

\section{Statistical Analysis}

Analyses were completed using SAS statistical software (SAS Institute, Inc., Cary, NC) and two-tailed tests of significance. First, univariate correlations assessed relationships between traditional PSG and spectral EEG indices and performance (ie, mean reaction time) on the SAT. In addition to TST and WASO, we selected 8 variables of interest due to prior links with cognition, including psychomotor functioning, and/or insomnia: relative delta, sigma, and beta power; ${ }^{10,65}$ sleep efficiency; SWS and REM percentages $;{ }^{51}$ and the initial peak of delta power and rate of decline in delta power overnight. ${ }^{19-32,41}$ Given the number of analyses performed, a Bonferroni correction was applied to adjust significance levels and account for multiple comparisons (cutoff set at $0.05 / 8=0.006$ ). Second, because our goal was to study parameters of sleep associated with psychomotor functioning that have not been previously well established (such as sleep duration and continuity), it was of interest to see if any parameters we might identify are related to TST and WASO. Thus, EEG variables that were significantly associated with psychomotor performance in the first set of analyses 
were identified, and univariate correlations evaluated relationships among these significant variables, TST, and WASO. These first two sets of analyses were conducted in the full sample as well as separately in each group (insomnia and HC).

Third, multivariable linear regressions controlling for sex, age, years of education, location of recording (home versus lab), WASO, and TST examined whether additional EEG variables linked with psychomotor functioning in the univariate correlations were associated with psychomotor performance in the full sample after accounting for shortened and fragmented sleep. We controlled for TST and WASO (a key measure of sleep maintenance) in these analyses to investigate unique associations of additional sleep factors to psychomotor functioning over and above the wellestablished contributions of sleep deprivation and continuity to cognition-related sleep restoration. To examine whether relationships between EEG variables and psychomotor performance were similar across groups, multivariable linear regressions were rerun with the addition of a group (insomnia versus HC) main effect as well as interaction terms (group by EEG variable of interest). Finally, exploratory univariate correlations examined whether similar EEG predictors were associated with performance (ie, mean reaction time) on commonly used tasks (SRT, CPT) as were correlated with performance on the challenging task (SAT).

\section{Results}

\section{Characteristics of the Sample}

Seventy-six individuals (37 with insomnia; $39 \mathrm{HC}$ ) from our prior study met the stricter AHI and PLMAI criteria and had sufficient spectral data to be included in the present analysis. Groups did not differ in $\operatorname{sex}\left(\chi^{2}(76)=0.21, p=0.65\right)$ nor age $(\mathrm{t}(74)=-1.87, p=0.07)$. As previously reported in the larger sample, ${ }^{15,41}$ individuals with insomnia included in the current analysis displayed greater WASO $(\mathrm{t}(74)=-2.39, p=0.02)$, lower initial delta power peak $(\mathrm{t}(74)=-3.03, p=$ $0.003)$, more gradual delta decline overnight $(\mathrm{t}(74)=-3.91, p<0.001)$, and slower reaction times on the SAT $(\mathrm{t}(74)=$ $-2.48, p=0.02)$ compared to healthy controls. See Table 1 for characteristics of the sample.

\section{Univariate Associations Between EEG Indices and Daytime Cognitive Performance}

In addition to WASO, only slower declines in overnight delta power and decreased initial delta power peak were associated with longer response latencies on the SAT in the full sample after applying a Bonferroni adjustment. Notably, decreased relative sigma power was associated with increased SAT reaction time prior to Bonferroni correction. Among adults with insomnia, more gradual overnight delta decline was the strongest predictor of longer SAT latency, followed by lower initial delta peak. Among HC, more gradual overnight delta decline was again the strongest predictor of longer SAT latency, followed by increased WASO and reduced relative delta power. See Table 2.

\section{Correlations of Overnight Delta Dynamics with TST and WASO in the Full Sample and by Group}

In the full sample, increased WASO was correlated with more gradual delta slope and lower delta peak, and a more gradual decline in overnight delta power was significantly associated with lower initial delta peak. When examined separately by group, increased WASO was associated with lower delta peak and more gradual delta decline for HC, but not for adults with insomnia. In contrast, greater TST was associated with more gradual delta decline for adults with insomnia, but not for HC. Slower delta decline was associated with lower delta peak in both groups. See Table 3.

\section{Multivariable Relationships of Overnight Delta Dynamics and Daytime Cognitive Performance}

In the full sample, more gradual delta decline was associated with longer response times on the SAT task after covarying for age, sex, education level, location, TST, and WASO (partial $r=0.39, \mathrm{~F}(7,68)=12.52, p<0.001$ ). See Figure 1 . Similarly, lower initial delta peak was associated with longer response times on the SAT task in the full sample after accounting for demographics, TST, and WASO (partial $r=-0.32, \mathrm{~F}(7,68)=7.85, p=0.007$ ). See Figure 2. Further, delta decline remained significantly related to SAT performance after controlling for initial delta peak (partial $r=0.25, \mathrm{~F}(8,67)$ $=4.37, p=0.04)$, suggesting the relationship between delta decline and SAT performance is not driven by the peak of 
Table I Characteristics of the Sample

\begin{tabular}{|c|c|c|}
\hline Demographic Characteristic & Insomnia $(n=37)$ & HC $(n=39)$ \\
\hline Age, mean (SD)* & $45.68(17.70)$ & $38.62(15.25)$ \\
\hline Sex, Female (\%) & $19(51 \%)$ & 21 (54\%) \\
\hline Education, Years, mean (SD) & $15.95(2.72)$ & $16.64(2.60)$ \\
\hline \multicolumn{3}{|l|}{ Race, number (\%) } \\
\hline Asian & $2(5 \%)$ & $2(5 \%)$ \\
\hline Black/African American & $7(19 \%)$ & $5(13 \%)$ \\
\hline Multiple Race Identity & I (3\%) & $2(5 \%)$ \\
\hline White/Caucasian & $27(73 \%)$ & $30(77 \%)$ \\
\hline \multicolumn{3}{|c|}{ Sleep Characteristic, mean (SD) } \\
\hline Relative Delta & $0.76(0.07)$ & $0.75(0.06)$ \\
\hline Relative Sigma & $0.18(0.06)$ & $0.19(0.05)$ \\
\hline Relative Beta & $-4.48(0.44)$ & $-4.54(0.35)$ \\
\hline SE (\%) & $0.84(0.09)$ & $0.87(0.13)$ \\
\hline WASO (Minutes)* & $49.66(38.97)$ & $31.83(27.99)$ \\
\hline TST (Minutes) & 377.91 (66.91) & $390.89(60.52)$ \\
\hline SWS \% & $0.20(0.10)$ & $0.21(0.09)$ \\
\hline REM \% & $0.20(0.04)$ & $0.21(0.06)$ \\
\hline Delta Decline* & $-1.17(1.99)$ & $-2.77(1.57)$ \\
\hline Delta Peak* & $1539.19(852.93)$ & $2163.28(936.7 I)$ \\
\hline \multicolumn{3}{|l|}{ Reaction Time, mean (SD) } \\
\hline SAT* & $439.08(137.56)$ & 374.54 (84.93) \\
\hline SRT & $239.26(74.13)$ & $213.56(39.31)$ \\
\hline CPT & $358.98(51.92)$ & $346.99(48.34)$ \\
\hline
\end{tabular}

Note: *Denotes statistically significant difference between groups, $p<0.05$.

Abbreviations: CPT, continuous performance task; REM, rapid eye movement; SAT, switching attention task; SRT, simple reaction task; SE, sleep efficiency; SWS, slow wave sleep; TST, total sleep time; WASO, wake after sleep onset.

Table 2 Correlations of EEG Sleep Variables and SAT Response Latency in the Full Sample

\begin{tabular}{|l|c|c|c|}
\hline & Full Sample & Insomnia & Healthy Controls \\
\hline WASO & $0.33^{* * *}$ & 0.22 & $0.41^{*}$ \\
TST & -0.15 & -0.16 & -0.08 \\
Relative Delta & 0.01 & 0.20 & $-0.36^{*}$ \\
Relative Sigma & $-0.23^{*}$ & $-0.28^{+}$ & -0.10 \\
Relative Beta & -0.02 & -0.12 & 0.10 \\
SE & -0.17 & -0.15 & -0.16 \\
SWS \% & -0.13 & -0.09 & -0.17 \\
REM \% & -0.01 & 0.06 & -0.01 \\
Delta Decline & $0.50^{* * *}$ & $0.42^{* *}$ & $0.48^{* * *}$ \\
Delta Peak & $-0.38^{* * *}$ & $-0.34^{*}$ & $-0.31^{+}$ \\
\hline
\end{tabular}

Notes: ***Indicates $p<0.006$ (Bonferroni cutoff), **Indicates $p<0.01$, *Indicates $p<0.05,{ }^{+}$Indicates $p<0.10$.

Abbreviations: REM, rapid eye movement; SE, sleep efficiency; SWS, slow wave sleep; TST, total sleep time; WASO, wake after sleep onset.

delta power at the beginning of the night. To further ensure that location of PSG recording did not impact observed relationships, we explored location of recording as a moderator and found that relationships between delta decline and peak and subsequent psychomotor performance did not differ by location of recording ( $p$ values for interaction terms were 0.89 and 0.73 , respectively). In addition, removal of two potential outliers who displayed more gradual delta decline 
Table 3 Correlations of Overnight Delta Dynamics with TST and WASO in the Full Sample and by Group

\begin{tabular}{|c|c|c|c|c|c|c|c|c|c|}
\hline & \multicolumn{3}{|c|}{ Full Sample } & \multicolumn{3}{|c|}{ Insomnia Group } & \multicolumn{3}{|c|}{ HC Group } \\
\hline & WASO & $\begin{array}{c}\text { Delta } \\
\text { Decline }\end{array}$ & $\begin{array}{c}\text { Delta } \\
\text { Peak }\end{array}$ & WASO & $\begin{array}{c}\text { Delta } \\
\text { Decline }\end{array}$ & $\begin{array}{l}\text { Delta } \\
\text { Peak }\end{array}$ & WASO & $\begin{array}{c}\text { Delta } \\
\text { Decline }\end{array}$ & $\begin{array}{l}\text { Delta } \\
\text { Peak }\end{array}$ \\
\hline TST & -0.17 & -0.21 & 0.02 & -0.20 & $-0.37^{*}$ & 0.20 & -0.01 & 0.06 & -0.22 \\
\hline WASO & - & $0.31 * *$ & $-0.31 * *$ & - & 0.15 & -0.19 & - & $0.36 *$ & $-0.32 *$ \\
\hline $\begin{array}{l}\text { Delta } \\
\text { Decline }\end{array}$ & - & - & $-0.74 * * *$ & - & - & $-0.63 * * *$ & - & - & $-0.80 * * *$ \\
\hline
\end{tabular}

Notes: ***Indicates $p<0.001$, **Indicates $p<0.01$, *Indicates $p<0.05$.

Abbreviations: TST, total sleep time; WASO, wake after sleep onset.

did not alter results for either the relationship of delta peak or delta slope to psychomotor performance, both of which remained significant ( $p$ values were 0.001 and 0.004 , respectively).

When group and group by delta decline interaction were added to the model, the main effect of delta decline on SAT reaction time remained significant $(\mathrm{F}(8,67)=12.52, p<0.001)$. Neither the group main effect $(\mathrm{F}(8,67)=0.24, p=0.63)$ nor group by delta decline interaction effect $(\mathrm{F}(9,66)=0.04, p=0.85)$ on SAT reaction time was significant, suggesting similar relationships between more gradual delta decline and slower reactions times across groups. Similarly, the main effect of initial delta peak remained significant in models including group and a group by delta peak interaction $(\mathrm{F}(8,67)$ $=6.02, p=0.02)$. Neither the main effect of group $(\mathrm{F}(8,67)=0.69, p=0.41)$ nor the interaction effect $(\mathrm{F}(9,66)=0.06$, $p=0.81)$ was significant, again suggesting similar relationships across groups.

\section{Exploratory Analyses}

Slower decline in delta power was the strongest predictor of longer reactions times on the SRT and CPT in the full sample. In addition, lower initial delta peak and greater WASO were associated with longer SRT latencies, and shorter TST and reduced SE were associated with longer CPT latencies in the full sample. See Table 4.

\section{Discussion}

The primary aim of the current study was to examine the relative contributions of PSG-derived sleep indices to psychomotor functioning (and, specifically, complex attention) and to identify the most prominent processes underlying sleep restoration related to cognitive function in adults with insomnia disorder and healthy controls. In addition to WASO, two PSG/EEG indices emerged as primary contributors to daytime psychomotor performance in our full sample after adjusting for multiple comparisons: initial delta peak and decline of delta power overnight. Interestingly, more gradual delta slope emerged as the strongest predictor of poor SAT performance in both the insomnia and healthy control groups. In addition, although the focus of the current study was on the most challenging cognitive test (SAT), exploratory analyses suggested associations between more gradual delta decline and longer latencies on commonly used cognitive tasks (CPT and SRT), suggesting this sleep factor may be generally supportive of psychomotor functioning, having effects observable even on less demanding tasks. It is notable that decreased sigma power was significantly correlated with poorer SAT performance prior to Bonferroni correction, which is consistent with prior studies linking sleep spindles and psychomotor functioning. ${ }^{42-50}$

We also examined overlap among the sleep indices supporting psychomotor functioning. In the full sample, WASO and measures of homeostatic sleep drive (delta decline and initial delta peak) were significantly associated, and these associations were generally stronger in the HC group. TST was also related to delta decline, but only for individuals with insomnia. Finally, multivariable regressions indicated that performance on the SAT was significantly associated with both homeostatic sleep drive measures (delta decline and delta peak) over and above shortened and fragmented sleep, and these relationships were similar across insomnia and healthy control groups.

Taken together, results of this study suggest a cognition-related sleep restoration process supported by several interrelated but distinct aspects of sleep: sleep continuity, recovery from and dissipation of homeostatic sleep drive, and, perhaps to a lesser extent, sigma power. These innovative results add to the existing literature in several ways. First, 


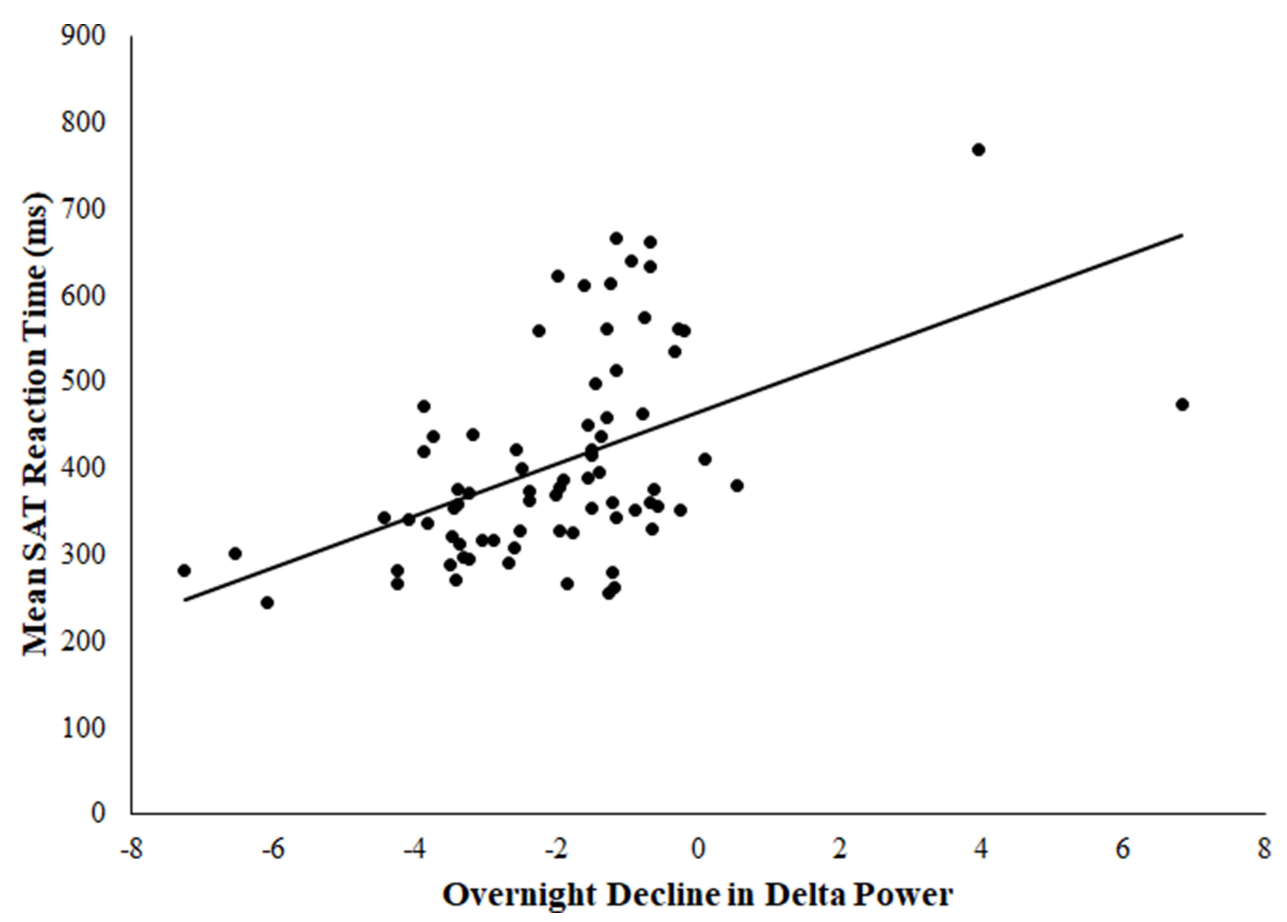

Figure I Association between overnight delta decline and SAT latency.

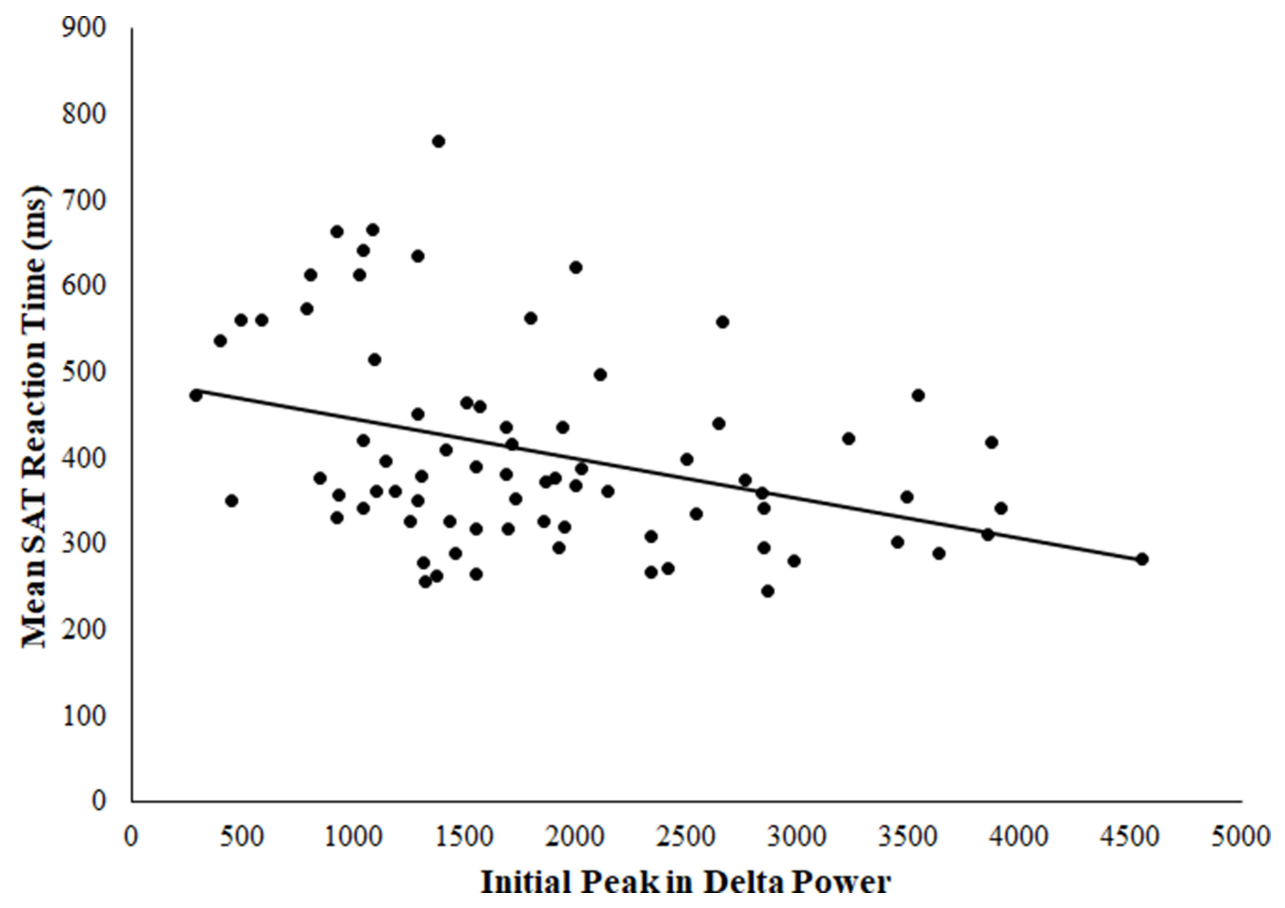

Figure 2 Association between initial delta peak and SAT latency.

these processes appear to impact daytime functioning in the absence of experimental sleep deprivation or reduced TST, suggesting they are integral to the restorative process of normal, unrestricted sleep. Second, similar patterns were observed in across groups, indicating that homeostatic sleep drive processes may be fundamental to supporting daytime psychomotor functioning in healthy individuals as well as in those with insomnia, suggesting broad societal importance. For example, common events that disrupt these sleep processes, such as caffeine use, ${ }^{71}$ alcohol use, ${ }^{72}$ and exposure to 
Table 4 Correlations of EEG Sleep Variables and SRT and CPT Response Latency in the Full Sample

\begin{tabular}{|l|c|c|}
\hline & SRT & CPT \\
\hline Relative Delta & 0.02 & 0.07 \\
Relative Sigma & -0.16 & -0.15 \\
Relative Beta & 0.03 & -0.10 \\
SE & -0.15 & $-0.24^{*}$ \\
WASO & $0.28^{*}$ & $0.22^{+}$ \\
TST & -0.04 & $-0.23^{*}$ \\
SWS \% & -0.02 & 0.10 \\
REM \% & -0.10 & -0.10 \\
Delta Decline & $0.32^{* *}$ & $0.32^{* *}$ \\
Delta Peak & $-0.28^{*}$ & -0.18 \\
\hline
\end{tabular}

Notes: **Indicates $p<0.01$, *Indicates $p<0.05$, ${ }^{+}$Indicates $p<0.10$.

Abbreviations: CPT, continuous performance task; REM, rapid eye movement; SRT, simple reaction task; SE, sleep efficiency; SWS, slow wave sleep; TST, total sleep time; WASO, wake after sleep onset.

stress, ${ }^{73}$ may contribute to increased cognitive dysfunction in the general population and may be reflected in delta dynamics measures.

In addition, many psychiatric disorders, including attention-deficit/hyperactivity disorder, depression, post-traumatic stress disorder, psychosis, and neurocognitive disorders such as dementia are characterized by both sleep problems ${ }^{74}$ and cognitive impairment, ${ }^{75}$ and future studies should examine whether disruptions to homeostatic sleep drive may represent a transdiagnostic process underlying psychomotor deficits across disorders. Relatedly, future studies should also examine whether insomnia treatments known to impact homeostatic sleep drive and its EEG markers (increased delta peak and steeper overnight decline), such as Cognitive-Behavioral Treatment for Insomnia (CBT-I), ${ }^{63}$ may improve psychomotor functioning among patients with insomnia and/or those presenting with a range of psychiatric disorders accompanied by sleep complaints. Finally, links between patterns of slow wave sleep (as reflected by overnight delta power dynamics) and psychomotor functioning, as shown in the current study, may be consistent with the synaptic homeostasis hypothesis (SHY), which suggests a role of sleep, and specifically slow wave activity, in supporting brain plasticity. ${ }^{76}$ Future research should examine if and how overnight delta dynamics, including delta decline, may be related to the synaptic homeostasis process.

This study is subject to limitations. First, the current study assessed only one aspect of cognition (psychomotor performance, and, more specifically, complex attention), and there is evidence to suggest that sleep disturbances impact a range of neurocognitive functions, such as memory, learning, risk-taking, and emotional reasoning. ${ }^{51}$ Future studies should investigate whether these same processes (recovery from and dissipation of homeostatic sleep drive) support performance across a range of neurocognitive tasks. Future work also should examine whether overnight delta dynamics are associated with measures related to sleep restoration other than psychomotor functioning, such as daytime sleepiness, mood, and cardiometabolic function. ${ }^{7,77}$ Second, the study sample was composed of research volunteers over the age 20, many of whom identified as White race. Thus, it is currently unclear whether similar results would be observed in more clinically severe individuals or younger (ie, children and adolescents) and more racially diverse samples. Third, spectral analysis was carried out using a central lead, and delta power tends to be greatest at frontal sites. ${ }^{78}$ Although our methods are consistent with our prior work investigating delta peak and decline in insomnia, ${ }^{10,41,63}$ it is possible that our approach may underestimate the association between delta power dynamics and psychomotor functioning. Future studies including frontal measures of delta power may evidence even larger effects than those observed in the current study. 


\section{Conclusion}

Although sleep duration and continuity may be important contributors to the sleep restoration process as it relates to psychomotor functioning, our findings suggest that processes associated with homeostatic sleep drive may represent factors underlying cognition-related restoration and daytime functioning in typical, unrestricted sleep. These data suggest these processes are more broadly relevant than just to the insomnia population, as similar findings were found in healthy controls; however, future work is needed to determine the extent to which these processes are universal. These results may have broad clinical implications for adults with cognitive impairments, and future research should focus on elucidating these mechanisms and enhancing interventions to improve homeostatic sleep drive and daytime functioning in adults.

\section{Acknowledgments}

We are grateful to the participants for their contributions to research.

\section{Author Contributions}

All authors made a significant contribution to the work reported, whether that is in the conception, study design, execution, acquisition of data, analysis and interpretation, or in all these areas; took part in drafting, revising, or critically reviewing the article; gave final approval of the version to be published; have agreed on the journal to which the article has been submitted; and agree to be accountable for all aspects of the work.

\section{Funding}

This work was supported by the National Institute of Mental Health Grants \# K23-MH-108704 awarded to Jessica R. Lunsford-Avery, Ph.D. and \# R01 MH076856 and \# R01 HL096492 to Dr. Krystal and the Department of Veterans Affairs Grant \# VA009 and the National Institute of Health Lung and Blood Grant \# R01-HL-073259-01 awarded to Dr. Edinger. Funding sponsors had no role in the study design or decision to submit this paper for publication.

\section{Disclosure}

Dr. Lunsford-Avery reports grants from NIMH during the conduct of the study; received honorarium for editing issue from Elsevier, outside the submitted work. Dr. Edinger receives consulting fees from Somly; also reports grants from Department of Veterans Affairs, grants from National Institutes of Health, during the conduct of the study. Dr. Krystal has received research support from Janssen Pharmaceuticals, Axsome Pharmaceutics, Reveal Biosensors, The Ray and Dagmar Dolby Family Fund, and the National Institutes of Health and consulting fees from Adare, Axsome Therapeutics, Big Health, Eisai, Evecxia, Ferring Pharmaceuticals, Galderma, Harmony Biosciences, Idorsia, Janssen Pharmaceuticals, Jazz Pharmaceuticals, Millennium Pharmaceuticals, Merck, Neurocrine Biosciences, Neurawell, Pernix, Otsuka Pharmaceuticals, Sage, Takeda, and Harmony. The authors report no other conflicts of interest in this work.

\section{References}

1. Walker MP. Cognitive consequences of sleep and sleep loss. Sleep Med. 2008;9(Suppl 1):S29-34. doi:10.1016/S1389-9457(08)70014-5

2. Lim J, Dinges DF. A meta-analysis of the impact of short-term sleep deprivation on cognitive variables. Psychol Bull. 2010;136(3):375-389. doi:10.1037/a0018883

3. Van Dongen HP, Maislin G, Mullington JM, Dinges DF. The cumulative cost of additional wakefulness: dose-response effects on neurobehavioral functions and sleep physiology from chronic sleep restriction and total sleep deprivation. Sleep. 2003;26(2):117-126. doi:10.1093/sleep/26.2.117

4. Frank MG. The mystery of sleep function: current perspectives and future directions. Rev Neurosci. 2006;17(4):375-392. doi:10.1515/ revneuro.2006.17.4.375

5. Stepanski EJ. The effect of sleep fragmentation on daytime function. Sleep. 2002;25(3):268-276. doi:10.1093/sleep/25.3.268

6. Roehrs T, Merlotti L, Petrucelli N, Stepanski E, Roth T. Experimental sleep fragmentation. Sleep. 1994;17(5):438-443. doi:10.1093/sleep/17.5.438

7. Bonnet MH. Sleep restoration as a function of periodic awakening, movement, or electroencephalographic change. Sleep. 1987;10(4):364-373. doi:10.1093/sleep/10.4.364

8. Ferri R, Drago V, Arico D, et al. The effects of experimental sleep fragmentation on cognitive processing. Sleep Med. 2010;11(4):378-385. doi:10.1016/j.sleep.2010.01.006

9. Cote KA, Milner CE, Osip SL, Ray LB, Baxter KD. Waking quantitative electroencephalogram and auditory event-related potentials following experimentally induced sleep fragmentation. Sleep. 2003;26(6):687-694. doi:10.1093/sleep/26.6.687 
10. Krystal AD, Edinger JD, Wohlgemuth WK, Marsh GR. NREM sleep EEG frequency spectral correlates of sleep complaints in primary insomnia subtypes. Sleep. 2002;25(6):630-640.

11. Arcia E, Otto DA. Reliability of selected tests from the neurobehavioral evaluation system. Neurotoxicol Teratol. 1992;14(2):103-110. doi:10.1016/ 0892-0362(92)90058-I

12. Baker EL, Letz R, Fidler A. A computer-administered neurobehavioral evaluation system for occupational and environmental epidemiology. Rationale, methodology, and pilot study results. J Occup Med. 1985;27(3):206-212.

13. Beck LH, Bransome ED Jr, Mirsky AF, Rosvold HE, Sarason I. A continuous performance test of brain damage. J Consult Psychol. 1956;20 (5):343-350. doi:10.1037/h0043220

14. Krystal AD. Treating the health, quality of life, and functional impairments in Insomnia. J Clin Sleep Med. 2007;3(1):63-72.

15. Edinger JD, Means MK, Carney CE, Krystal AD. Psychomotor performance deficits and their relation to prior nights' sleep among individuals with primary insomnia. Sleep. 2008;31(5):599-607. doi:10.1093/sleep/31.5.599

16. Van Someren EJW. Brain mechanisms of insomnia: new perspectives on causes and consequences. Physiol Rev. 2021;101(3):995-1046. doi:10.1152/physrev.00046.2019

17. Perrier J, Chavoix C, Bocca ML. Functioning of the three attentional networks and vigilance in primary insomnia. Sleep Med. 2015;16 (12):1569-1575. doi:10.1016/j.sleep.2015.06.025

18. Ballesio A, Aquino M, Kyle SD, Ferlazzo F, Lombardo C. Executive functions in insomnia disorder: a systematic review and exploratory meta-analysis. Front Psychol. 2019;10:101. doi:10.3389/fpsyg.2019.00101

19. Achermann P, Dijk DJ, Brunner DP, Borbely AA. A model of human sleep homeostasis based on EEG slow-wave activity: quantitative comparison of data and simulations. Brain Res Bull. 1993;31(1-2):97-113. doi:10.1016/0361-9230(93)90016-5

20. Werth E, Dijk DJ, Achermann P, Borbely AA. Dynamics of the sleep EEG after an early evening nap: experimental data and simulations. Am J Physiol Regul Integr Comp Physiol. 1996;271(3):R501-R510. doi:10.1152/ajpregu.1996.271.3.R501

21. Franken P, Dijk DJ, Tobler I, Borbely AA. Sleep-deprivation in rats - effects on eeg power spectra, vigilance states, and cortical temperature. $A m$ J Physiol. 1991;261(1):R198-R208.

22. Dijk DJ, Hayes B, Czeisler CA. Dynamics of electroencephalographic sleep spindles and slow wave activity in men: effect of sleep deprivation. Brain Res. 1993;626(1-2):190-199. doi:10.1016/0006-8993(93)90579-C

23. Dijk DJ, Cajochen C, Tobler I, Borbely AA. Sleep extension in humans: sleep stages, EEG power spectra and body temperature. Sleep. 1991;14 (4):294-306. doi:10.1093/sleep/14.4.294

24. Dijk DJ, Brunner DP, Borbely AA. Time course of EEG power density during long sleep in humans. Am J Physiol. 1990;258(3 Pt 2):R650-661. doi:10.1152/ajpregu.1990.258.3.R650

25. Dijk DJ, Brunner DP, Borbely AA. Eeg power-density during recovery sleep in the morning. Electroencephalogr Clin Neurophysiol. 1991;78 (3):203-214. doi:10.1016/0013-4694(91)90034-2

26. Dijk DJ, Brunner DP, Beersma DG, Borbely AA. Electroencephalogram power density and slow wave sleep as a function of prior waking and circadian phase. Sleep. 1990;13(5):430-440. doi:10.1093/sleep/13.5.430

27. Dijk DJ. EEG slow waves and sleep spindles: windows on the sleeping brain. Behav Brain Res. 1995;69(1-2):109-116. doi:10.1016/0166-4328(95) $00007-\mathrm{G}$

28. Brunner DP, Dijk DJ, Tobler I, Borbely AA. Effect of partial sleep-deprivation on sleep stages and eeg power spectra - evidence for non-rem and rem-sleep homeostasis. Electroencephalogr Clin Neurophysiol. 1990;75(6):492-499. doi:10.1016/0013-4694(90)90136-8

29. Brunner DP, Dijk DJ, Borbely AA. Repeated partial sleep-deprivation progressively changes the eeg during sleep and wakefulness. Sleep. 1993;16 (2):100-113. doi:10.1093/sleep/16.2.100

30. Tobler I, Borbely AA. Sleep eeg in the rat as a function of prior waking. Electroencephalogr Clin Neurophysiol. 1986;64(1):74-76. doi:10.1016/ 0013-4694(86)90044-1

31. Borbely AA, Baumann F, Brandeis D, Strauch I, Lehmann D. Sleep-deprivation - effect on sleep stages and eeg power-density in man. Electroencephalogr Clin Neurophysiol. 1981;51(5):483-493. doi:10.1016/0013-4694(81)90225-X

32. Endo T, Schwierin B, Borbely AA, Tobler I. Selective and total sleep deprivation: effect on the sleep EEG in the rat. Psychiat Res. 1997;66(23):97-110. doi:10.1016/S0165-1781(96)03029-6

33. Esser SK, Hill SL, Tononi G. Sleep homeostasis and cortical synchronization: i. Modeling the effects of synaptic strength on sleep slow waves. Sleep. 2007;30(12):1617-1630. doi:10.1093/sleep/30.12.1617

34. Vyazovskiy VV, Cirelli C, Tononi G. Electrophysiological correlates of sleep homeostasis in freely behaving rats. Prog Brain Res. 2011;193:17-38.

35. Bjorness TE, Dale N, Mettlach G, et al. An adenosine-mediated glial-neuronal circuit for homeostatic sleep. J Neurosci. 2016;36(13):3709-3721. doi:10.1523/JNEUROSCI.3906-15.2016

36. Bjorness TE, Kelly CL, Gao T, Poffenberger V, Greene RW. Control and function of the homeostatic sleep response by adenosine A1 receptors. J Neurosci. 2009;29(5):1267-1276. doi:10.1523/JNEUROSCI.2942-08.2009

37. Greene RW, Bjorness TE, Suzuki A. The adenosine-mediated, neuronal-glial, homeostatic sleep response. Curr Opin Neurobiol. 2017 ;44:236-242. doi:10.1016/j.conb.2017.05.015

38. Halassa MM, Florian C, Fellin T, et al. Astrocytic modulation of sleep homeostasis and cognitive consequences of sleep loss. Neuron. 2009;61 (2):213-219. doi:10.1016/j.neuron.2008.11.024

39. Landolt HP. Sleep homeostasis: a role for adenosine in humans? Biochem Pharmacol. 2008;75(11):2070-2079. doi:10.1016/j.bcp.2008.02.024

40. Retey JV, Adam M, Honegger E, et al. A functional genetic variation of adenosine deaminase affects the duration and intensity of deep sleep in humans. Proc Natl Acad Sci U S A. 2005;102(43):15676-15681. doi:10.1073/pnas.0505414102

41. Lunsford-Avery JR, Edinger JD, Krystal AD. Optimizing computation of overnight decline in delta power: evidence for slower rate of decline in delta power in insomnia patients. Clin Neurophysiol. 2021;132(2):545-553. doi:10.1016/j.clinph.2020.12.004

42. Mednick SC, McDevitt EA, Walsh JK, et al. The critical role of sleep spindles in hippocampal-dependent memory: a pharmacology study. J Neurosci. 2013;33(10):4494-4504. doi:10.1523/JNEUROSCI.3127-12.2013

43. Fogel SM, Smith CT. The function of the sleep spindle: a physiological index of intelligence and a mechanism for sleep-dependent memory consolidation. Neurosci Biobehav Rev. 2011;35(5):1154-1165. doi:10.1016/j.neubiorev.2010.12.003 
44. Lafortune M, Gagnon JF, Martin N, et al. Sleep spindles and rapid eye movement sleep as predictors of next morning cognitive performance in healthy middle-aged and older participants. J Sleep Res. 2014;23(2):159-167. doi:10.1111/jsr.12108

45. Wamsley EJ, Shinn AK, Tucker MA, et al. The effects of eszopiclone on sleep spindles and memory consolidation in schizophrenia: a randomized placebo-controlled trial. Sleep. 2013;36(9):1369-1376. doi:10.5665/sleep.2968

46. Chatburn A, Coussens S, Lushington K, Kennedy D, Baumert M, Kohler M. Sleep spindle activity and cognitive performance in healthy children. Sleep. 2013;36(2):237-243. doi:10.5665/sleep.2380

47. Lustenberger C, Maric A, Durr R, Achermann P, Huber R. Triangular relationship between plos one spindle activity, general cognitive ability and the efficiency of declarative learning. PLoS One. 2012;7(11):294-306. doi:10.1371/journal.pone.0049561

48. Barakat M, Carrier J, Debas K, et al. Sleep spindles predict neural and behavioral changes in motor sequence consolidation. Hum Brain Mapp. 2013;34(11):2918-2928. doi:10.1002/hbm.22116

49. Schabus M, Hoedlmoser K, Pecherstorfer T, et al. Interindividual sleep spindle differences and their relation to learning-related enhancements. Brain Res. 2008;1191:127-135. doi:10.1016/j.brainres.2007.10.106

50. Schabus M, Hodlmoser K, Gruber G, et al. Sleep spindle-related activity in the human EEG and its relation to general cognitive and learning abilities. Eur J Neurosci. 2006;23(7):1738-1746. doi:10.1111/j.1460-9568.2006.04694.x

51. Della Monica C, Johnsen S, Atzori G, Groeger JA, Dijk DJ. Rapid eye movement sleep, sleep continuity and slow wave sleep as predictors of cognition, mood, and subjective sleep quality in healthy men and women, aged 20-84 years. Front Psychiatry. 2018;9. doi:10.3389/ fpsyt.2018.00255

52. Edinger JD, Means MK, Krystal AD. Does physiological hyperarousal enhance error rates among insomnia sufferers? Sleep. 2013;36 (8):1179-1186. doi:10.5665/sleep.2882

53. Edinger JD, Glenn DM, Bastian LA, et al. Sleep in the laboratory and sleep at home II: comparisons of middle-aged insomnia sufferers and normal sleepers. Sleep. 2001;24(7):761-770. doi:10.1093/sleep/24.7.761

54. Edinger JD, Fins AI, Sullivan RJ, et al. Sleep in the laboratory and sleep at home: comparisons of older insomniacs and normal sleepers. Sleep. 1997;20(12):1119-1126. doi:10.1093/sleep/20.12.1119

55. First M, Spitzer RL, Gibbon M, Williams JB. Structured Clinical Interview for the DSM-IV Axis I Disorders (SCID-I), Patient Edition. Washington, D.C.: American Psychiatric Press; 1995.

56. Schramm E, Hohagen F, Grasshoff U, et al. Test-retest reliability and validity of the structured interview for sleep disorders according to DSM-IIIR. Am J Psychiat. 1993;150(6):867-872.

57. Edinger JD, Bonnet MH, Bootzin RR, et al. Derivation of research diagnostic criteria for insomnia: report of an American Academy of Sleep Medicine Work Group. Sleep. 2004;27(8):1567-1596. doi:10.1093/sleep/27.8.1567

58. Bubu OM, Andrade AG, Umasabor-Bubu OQ, et al. Obstructive sleep apnea, cognition and Alzheimer's disease: a systematic review integrating three decades of multidisciplinary research. Sleep Med Rev. 2020;50:101250.

59. Leng Y, Blackwell T, Stone KL, Hoang TD, Redline S, Yaffe K. Periodic limb movements in sleep are associated with greater cognitive decline in older men without dementia. Sleep. 2016;39(10):1807-1810. doi:10.5665/sleep.6158

60. Edinger JD, Marsh GR, Mccall WV, Erwin CW, Lininger AW. Sleep variability across consecutive nights of home monitoring in older mixed dims patients. Sleep. 1991;14(1):13-17. doi:10.1093/sleep/14.1.13

61. Edinger JD, Hoelscher TJ, Webb MD, Marsh GR, Radtke RA, Erwin CW. Polysomnographic assessment of dims - empirical-evaluation of its diagnostic-value. Sleep. 1989;12(4):315-322.

62. Rechtshaffen A, Kales A. A Manual of Standardized Terminology, Technique, and Scoring System for Sleep Stages of Human Sleep. Los Angeles, CA: Brain Information Service; 1968.

63. Krystal AD, Edinger JD. Sleep EEG predictors and correlates of the response to cognitive behavioral therapy for insomnia. Sleep. $2010 ; 33$ (5):669-677. doi:10.1093/sleep/33.5.669

64. Krystal AD. Non-REM sleep EEG spectral analysis in insomnia. Psychiat Ann. 2008;38(9):615.

65. Perlis ML, Smith MT, Andrews PJ, Orff H, Giles DE. Beta/Gamma EEG activity in patients with primary and secondary insomnia and good sleeper controls. Sleep. 2001;24(1):110-117. doi:10.1093/sleep/24.1.110

66. Merica H, Blois R, Gaillard JM. Spectral characteristics of sleep EEG in chronic insomnia. Eur J Neurosci. 1998;10(5):1826-1834. doi:10.1046/ j.1460-9568.1998.00189.x

67. Buysse DJ, Germain A, Hall ML, et al. EEG spectral analysis in primary insomnia: NREM period effects and sex differences. Sleep. 2008;31 (12):1673-1682. doi:10.1093/sleep/31.12.1673

68. Wohlgemuth WK, Edinger JD, Fins AI, Sullivan RJ. How many nights are enough? The short-term stability of sleep parameters in elderly insomniacs and normal sleepers. Psychophysiology. 1999;36(2):233-244. doi:10.1111/1469-8986.3620233

69. Letz R, Baker EL. NES2 Neurobehavioral Evaluation System. Winchester, MA: Neurobehavioral Evaluation System; 1988.

70. Mahoney FC, Moore PA, Baker EL, Letz R. Experimental nitrous oxide exposure as a model system for evaluating neurobehavioral tests. Toxicology. 1988;49(2-3):449-457. doi:10.1016/0300-483X(88)90031-5

71. Landolt HP, Retey JV, Tonz K, et al. Caffeine attenuates waking and sleep electroencephalographic markers of sleep homeostasis in humans. Neuropsychopharmacol. 2004;29(10):1933-1939. doi:10.1038/sj.npp.1300526

72. Thakkar MM, Sharma R, Sahota P. Alcohol disrupts sleep homeostasis. Alcohol. 2015;49(4):299-310. doi:10.1016/j.alcohol.2014.07.019

73. Saper CB, Cano G, Scammell TE. Homeostatic, circadian, and emotional regulation of sleep. J Comp Neurol. 2005;493(1):92-98. doi:10.1002/ cne. 20770

74. Freeman D, Sheaves B, Waite F, Harvey AG, Harrison PJ. Sleep disturbance and psychiatric disorders. Lancet Psychiat. $2020 ; 7(7): 628-637$. doi:10.1016/S2215-0366(20)30136-X

75. Millan MJ, Agid Y, Brune M, et al. Cognitive dysfunction in psychiatric disorders: characteristics, causes and the quest for improved therapy. Nat Rev Drug Discov. 2012;11(2):141-168. doi:10.1038/nrd3628

76. Tononi G, Cirelli C. Sleep and the price of plasticity: from synaptic and cellular homeostasis to memory consolidation and integration. Neuron. 2014;81(1):12-34. doi:10.1016/j.neuron.2013.12.025

77. Bonnet MH, Arand DL. Insomnia, metabolic rate and sleep restoration. J Intern Med. 2003;254(1):23-31. doi:10.1046/j.1365-2796.2003.01176.x 
78. Bernardi G, Betta M, Ricciardi E, Pietrini P, Tononi G, Siclari F. Regional delta waves in human rapid eye movement sleep. J Neurosci. 2019;39 (14):2686-2697. doi:10.1523/JNEUROSCI.2298-18.2019

\section{Publish your work in this journal}

Nature and Science of Sleep is an international, peer-reviewed, open access journal covering all aspects of sleep science and sleep medicine, including the neurophysiology and functions of sleep, the genetics of sleep, sleep and society, biological rhythms, dreaming, sleep disorders and therapy, and strategies to optimize healthy sleep. The manuscript management system is completely online and includes a very quick and fair peer-review system, which is all easy to use. Visit http://www.dovepress.com/testimonials.php to read real quotes from published authors.

Submit your manuscript here: https://www.dovepress.com/nature-and-science-of-sleep-journal 